\title{
Sachwortverzeichnis/Subject Index 1990
}

A

Adenylzyklase, 301 Adnextumor, 268 Adriamycin, 24,194 -/DTIC448 -/Ifosfamide, 448

Ätherlipidanaloga, 295 Alkylphosphocholine, 295 Alopezie, 24 Alpha-Interferon, 117, 217 Anämie

- $\quad$ tumorassoziierte

chronische, 46

Antagonisten, 5-HT3, 369 Antibiotika, 38 Antiemese, 313 Antiemetika, 369 Antigestagene, 237

Antimykotika, 38 Antiöstrogene, 237 Antitumoraktivität, 175 Antitumor-Substanzen,

- experimentelle, 245 Aromataseinhibitoren,

237

B

Bioethik, 85 Biometrische Planung und

Auswertung, 90 Bioverfügbarkeit, 203 Bronchialkarzinom, 144,

285

kleinzelliges, 141, 157, 253

nicht-kleinzelliges, 180, 186

$\mathrm{C}$

Calcitonin, 198 Carboplatin, 190 Chemo-Radiotherapie,

132 Chemotherapie, 85,186,

$198,221,237,253,338$,

424 -adjuvante, 24

- $\quad$ alternierende, 157

Cisplatin, 194, 310, 364

-undBestrahlung, 144

CMF-Regime, 203

COPBIAM, 132

Cyclophosphamid, 203

Cytosin-Arabinosid, 33

D

Diabetes mellitus, 17 Dialysierbarkeit, 289 DNA Fingerprinting, 305 DNS-Synthese, 453

Dosiseskalation, 50,190

- pharmakokinetisch

geleitete, 90

Dosisintensität, 132, 272 Doxorubicin-Derivat, 175

$\mathrm{E}$

Emesis, 364

Epidoxorubicin, 50, 272

Epirubicin, 24,141,313

Erhaltungsbehandlung, 
458 Erythropoetin, 46 Etherlipide, 245 Ethik, - medizinische, 85 Etoposid, 194 Evaluation, 375 $\mathrm{F}$

FAB-Klassifikation, 280 Fallzahl, 90 FAMTX-Schema, 354 Ferritin, 102 Fibonaci-Schema, - modifiziertes, 90 Fieber, 38 Fluorouracil, 50, 437, 453,465 Forschung, - klinische, 85 Fotemustin, 7

$\mathrm{G}$

Ganzabdomen-

bestrahlung, 260 GM-CSF, 33 G-Protein, 301 Gewebetoxizität, 346 Gliom, 305 Gynäkomastie, 128

$\mathrm{H}$

Hämodialyse, 289 Hals- und Kopftumor, 310 Hormontherapie, 237 Hyaluronidase, 310

Hyperinsulinismus, 17 Hypernephrom, 217

I

Immunologische Marker,

166 Immunstimulation,

präoperative, 124 Immuntherapie, adoptive,

416 Induktionstherapie, 43,

458 Informationsdienste im

Gesundheitswesen, 375 Informationssuche als

Coping-Verhalten, 375 Infusion,

-kontinuierliche, 117 ,

429 Insertionsmutagenese,

405 Interferon, 137, 424 Interleukin, 137, 416 -natiirliches, 429 Intoxikation, 289 In-vitro Test-

System, 462

$\mathrm{J}$

Jodo-Doxorubicin, 346

$\mathrm{K}$

Kardiotoxizität, 346, 465 Karzinoembryonales

Antigen, 198 Klinische Pharmakologie,

346 Knochenmarkkarzinose,

317 Kolonkarzinom-Zelliníe,

437 Kolorektalkarzinom, 21,

444 Kombinationschemo-

therapie, 28,194, 359 Koronarthrombose, 221 Krebsinformationsdienst, 375

L

Langzeittherapie, 313 Lebensqualität, 85 Leberfunktion, 381 Leberperfusion, hypertherme, 381 Leukämie, 38

akute lymphoblastische, 166

akute myeloische, 280

chronisch lymphatische, 424

chronisch myeloische, 109

Leukämiezellen,

residuale, 166 Leukopenie, 24 Leukovorin, 453 LH-RH-Antagonisten , 237 Lymphom, malignes, 102

$\mathrm{M}$ 
Magenkarzinom, 124,

194,354 Mammakarzinom, 17, 24,

$175,207,210,237,338$

- $\quad$ beim Mann, 128

- $\quad$ metastasierendes, 272

MCA, 210

Megestrolacetat, 285

Melanom, malignes, 7 ,

137 Melphalan (Alkeran), 96 Membrantoxizität, 245 Metabolisierung, 346 Metastasen, 24, 338

Metastasierung, 17 Mitomycin C, 50 Mitoxantron, 24 Monochemotherapie, 180

MorbusHodgkin, 132,

221 Multilocus-Sonden,

hypervariable, 305 Mycosis fungoides, 424

Myelodysplasie, 33 Myelom, multiples, 43, 46 Myelosuppression, 175 Myokardinfarkt, 465

$\mathrm{N}$

Nacktmaus, 305 Neoplasie Пa, multiple

endokrine, 317 Neuroblastom, 12 -zellen,301 Neutropenie, 38 Niereninsuffizienz, 289

Nierenzellkarzinom, 137,

217, 359 Nitroso-Harnstoffe, 7 Non-Hodgkin-Lym-

phome, 424

- $\quad$ hochmaligne, 28

Nukleotide, zyklische, 12

$\mathrm{O}$

Ösophaguskarzinom Ondansetron, 313, 369 Onkogenese, 405 Ovarialkarzinom, 260

$\mathrm{P}$

Pankreaskarzinom, fortgeschrittenes, 50

PDE, 12

Peritonealkrazinose, 388

Pharmakokinetik, 117, 346

Phase I, 346

- $\quad$ Studie, 429

Phase II

-Prüfung, 190

-Studie, 444

Photodynamische

Therapie, 462 PhotosanПI,462 Pirarubicin, 175,180 Postmenopause, 268 Postoperative Komplikationen, 124 Präklinisches Profil, 346 Prognostische Faktoren,

$109,144,237$

- $\quad$ Untergruppen, 338

Propionibacterium

avidum KP-40,124 Prostatakarzinom, 388 -kleinzelliges, 388

$\mathrm{R}$

Radiotherapie, 28 Raji Zellen, 295 Retro viren, 405 Risikofaktoren, 128 Risikogruppen, 260

$\mathrm{S}$

Schilddrüsenkarzinom, medull-äres, 198,317 Screening, 268

Sensitivität, 210 Serotoninantagonisten, 
364 Singulett Sauerstoff, 462 Spezifität, 210 Standard-Prognose-

Modell Steroid-Hormonrezeptor,

21 Stoppregel, 90 Strahlentherapie, 253, 310 Studien

frühe klinische, 245

multizentrische, 28

Phase I, 85

randomisierte, 253

retrospektive, 280 Subkutan, 117 Supportive Therapie, 369

$\mathrm{T}$

Tauromustin, 186 Ternärer Komplex, 453 Thymidylat-Synthase, 453 Toleranz, 117 Toxizität, 96

Trans-Aktivierung, 405 Transduktion, 405 Triphosphate, 12 Tumor-

-größe, initiale, 144 -kachexie, 285 -marker, 102,207,210

- $\quad$ nekrosefaktor, 285, 444

-stadium, 102

$\mathrm{U}$

Überdosierung, 96, 289 Überlebensrate, 124 Übersichtsarbeit, 157 Ultraschall, 268

$\mathrm{V}$

Vaginosonographie, 268 Versuche am Menschen, 85 Vindesin, 310 -undBestrahlung, 144

W

Wachstumsf aktoren,

hämatopoetische, 33,

437 Wachstumshemmung, 295 Wachstumsmodulation,

437 Weichteilsarkome,

metastasierte, 448

$\mathrm{Z}$

Zelldifferénzierung, 12 Zellinienstabilität, 305 Zentrale Erfassung, 128 Zinkkonzentration im

Plasma, 207 Zytokine, 416 Zytostatika, 289, 369 Zytotoxízität,295,346 